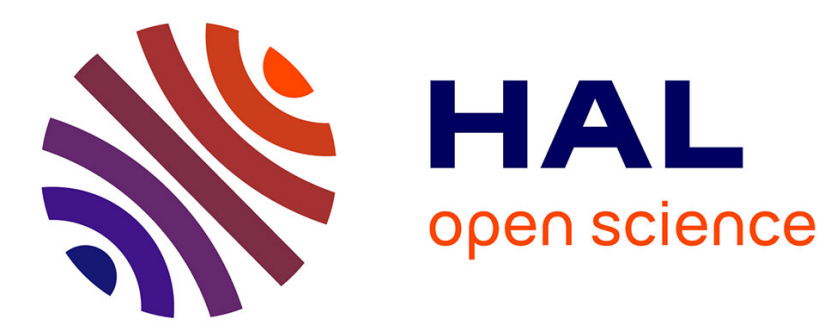

\title{
Determination of the wettability of powders by the Washburn capillary rise method with bed preparation by a centrifugal packing technique
}

\author{
Laurence Galet, Severine Patry, John A. Dodds
}

\section{To cite this version:}

Laurence Galet, Severine Patry, John A. Dodds. Determination of the wettability of powders by the Washburn capillary rise method with bed preparation by a centrifugal packing technique. Journal of Colloid and Interface Science, 2010, 346 (2), p. 470-475. 10.1016/j.jcis.2010.02.051 . hal-01593332

\section{HAL Id: hal-01593332 \\ https://hal.science/hal-01593332}

Submitted on 26 Apr 2019

HAL is a multi-disciplinary open access archive for the deposit and dissemination of scientific research documents, whether they are published or not. The documents may come from teaching and research institutions in France or abroad, or from public or private research centers.
L'archive ouverte pluridisciplinaire HAL, est destinée au dépôt et à la diffusion de documents scientifiques de niveau recherche, publiés ou non, émanant des établissements d'enseignement et de recherche français ou étrangers, des laboratoires publics ou privés. 


\title{
Determination of the wettability of powders by the Washburn capillary rise method with bed preparation by a centrifugal packing technique
}

\author{
Laurence Galet *, Severine Patry, John Dodds \\ Ecole des Mines d'Albi, Centre RAPSODEE, F-81013 Albi, France \\ Université de Toulouse, Mines Albi, CNRS, F-81013 Albi, France
}

\section{A B S T R A C T}

The Washburn capillary rise method is a standard technique for determining the wettability of powders expressed as a contact angle. The method requires the preparation of two identical beds of powder. One of these beds is used to follow the capillary rise with a perfectly wetting liquid (contact angle $=0$ ) giving access to a bed structure parameter. The other bed is used with the liquid of interest (contact angle $\neq 0$ ) and the capillary rise data is analysed using the previously determined structure parameter to obtain the contact angle. In the experiments reported here we have used a centrifugal packing technique to prepare beds of powder. This gives reproducible packings and also allows a certain degree of control of the bed porosity. In addition the air permeability of the beds is also determined prior to the capillary rise experiments. The results show that the value of the contact angle of a powder determined by the Washburn method depends on the porosity of the powder bed, and that the structure parameter can be determined from the air permeability using the Kozeny-Carman expression.

Keywords:

Washburn method

Capillary rise

Contact angle

Permeability

Porosity

\section{Introduction}

The wettability of a powder is an important property involved in many practical problems. For example, characterising the dispersability of a powder in a liquid, evaluating the bioavailability of a medicine, selecting a liquid binder to granulate a powder, etc. As illustrated in Fig. 1, a measure of the wettability of a solid is given by the contact angle $(\theta)$ as defined by Young equation [1] (Eq. (1)) of the force balance at the three-phase interface: solid $(s)$, liquid $(l)$, vapour $(v)$.

$\cos \theta=\frac{\gamma_{s v}-\gamma_{s l}}{\gamma_{l v}}$

This angle can be determined directly by measurements of the shape of a drop of liquid laid on a smooth solid surface. With solids in the form of powder it is possible to make a similar measurement by placing the liquid drop on a free or a compacted powder [2] but such a surface cannot be smooth at a particle level thus bringing into doubt the measured contact angle. Also, if the liquid wets the compacted powder, the drop will be imbibed by capillarity inside the powder compact making practical measurement difficult, even impossible.

Other methods for determining the wettability of a powder involve less direct and more complicated techniques: immersion cal-

\footnotetext{
* Corresponding author. Address: Ecole des Mines d'Albi, Centre RAPSODEE, F81013 Albi, France. Fax: +33 563493243.

E-mail address: laurence.galet@mines-albi.fr (L. Galet).
}

orimetry, inverse gas chromatography (IGC), dynamic vapour sorption isotherms (DVS). For a given powder-liquid system there are often significant differences in values obtained by these different techniques, as they do not all relate to the same definition of wettability. The only simple direct techniques applicable to powder samples are by those using capillary suction when a wetting liquid contacts a powder. Of these there are two main types: static tests and dynamic tests.

\section{Contact angles for powders by static tests}

Static tests for determining the contact angle are based on the Laplace equation for capillary rise in a tube [3].

$$
P_{\text {capillary }}=h_{\infty} \rho_{l} g=\frac{4}{D_{\text {tube }}} \gamma_{l} \cos \theta
$$

Here $P_{\text {capillary }}$ is the pressure due to capillary forces acting in a tube of diameter $D_{\text {tube }}$. Therefore measuring $\left(h_{\infty}\right)$ the final height to which a liquid of known density $\left(\rho_{l}\right)$ and surface tension $\left(\gamma_{l}\right)$ rises in the tube can be used to determine the contact angle. It has been shown by Dodds and Srivastava [4] that this expression can be modified to apply to a bed of powder based on the mean hydraulic diameter determined by the porosity $(\varepsilon)$, the specific surface of the particles $\left(S_{0}\right)$ or the surface mean diameter of the particles $\left(D_{s v}\right)$, Eq. (3).

$P_{\text {capillary }}=h_{\infty} \rho_{l} g=\frac{6(1-\varepsilon)}{D_{s \nu} \varepsilon} \gamma_{l} \cos \theta$ 

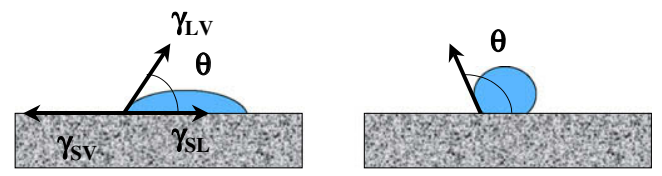

Fig. 1. The wettability of a solid by the contact angle.

Thus the contact angle can be determined by measuring the equilibrium height of capillary rise $\left(h_{\infty}\right)$ in a bed of powder, or by measuring the pressure required to displace a liquid from a bed of powder $\left(P_{\text {capillary }}\right)$. The first of these methods is not really practical as for a powder of say $50 \mu \mathrm{m}$ mean particle size, the capillary height of water would be of the order of $1 \mathrm{~m}$ thus requiring a rather large sample and a long time to reach equilibrium, perhaps even several months [5]. The second method can be used but requires purpose built equipment, not commercially available.

\section{Contact angle in powders by the Washburn method}

The contact angle at a powder can be determined from the dynamics of capillary flow. Bell and Cameron [6] showed experimentally that when a liquid penetrates in a tube by capillarity there is a linear relation between the square of the length of penetration and the time. Washburn made a theoretical analysis of capillarity driven Poiseuille flow of liquid in horizontal or vertical capillary tubes and, neglecting the effects due to the initial transient flow regime and the flow of displaced air, derived the differential Eq. (4a) of which the solution is given by Eq. (4b) [7].

$\frac{d l}{d t}=\frac{D_{\text {tube }}}{\eta} \frac{\gamma}{8} \cos \theta$

$\frac{l^{2}}{t}=\frac{D_{\text {tube }}}{4} \frac{\gamma}{\eta} \cos \theta$

Thus a plot of the square of the length of liquid penetration in a bed $\left(l^{2}\right)$ versus time $(t)$ gives a straight line, the slope of which is Bell and Cameron's constant, now identified as depending on both on the geometry of the capillary tube (diameter), the properties of the liquid (viscosity; $\eta$ and surface tension; $\gamma$ ) and also on the interaction between the solid and the liquid expressed by the contact angle. More recently, Xiao et al. [8] have presented a unified non-linear second-order differential equation for the problem taking into account inertial forces and entrance effects. An analytical solution was obtained, however comparison was not made with the simple $l^{2} / t$ relation and it is difficult to identify simply the role of the basic parameters. Fries and Dreyer [9] have given another analytical solution for capillary rise. They show that in practice the simple Washburn equation can be used with a precision of $3.7 \%$ for capillary rise of up to $10 \%$ of $h_{\infty}$.

Capillary rise is used in practice to determine the contact angle of a powder by what has become to be known as Washburn's method. A powder packed into a cylindrical cell with a porous bottom is touched on a liquid and, as the liquid penetrates the powder bed by capillarity, the increase in weight $(M)$ with time is followed. The weight of liquid in the sample tube is given by Eq. (5), which substituted into $(4 \mathrm{~b})$ gives $(6 a)$ and $(6 b)$.

$M=\frac{\pi D_{\text {tube }}^{2} l \rho_{l}}{4}$

$\frac{M^{2}}{t}=\frac{\pi^{2} D_{\text {tube }}^{5}}{64} \frac{\rho_{l}^{2} \gamma}{\eta} \cos \theta=C_{w} \frac{\rho_{l}^{2} \gamma}{\eta} \cos \theta$

The constant $C_{w}$ only depends on the "geometry" of the powder bed. A first experiment is performed with a liquid that is known to fully wet the powder (known $\rho_{l}$ and $\gamma$ with $\cos \theta=1$ ) thus allowing determination of the constant $C_{w}$. A second experiment is then performed using the liquid under study (also of known $\rho_{l}$ and $\gamma$ but with $\cos \theta \neq 1$ ) using the same powder packed in the same way (therefore the same $C_{w}$ ). This allows determining the contact angle of that liquid with the powder. According to the analysis of Fries and Dreyer [9], for particles as big as $100 \mu \mathrm{m}$ a typical sample of $5 \mathrm{~cm}$ length will ensure a precision of $3.7 \%$, and a precision of better than $1 \%$ can be obtained for particles smaller than $20 \mu \mathrm{m}$.

The validity of the method depends on three main assumptions. Firstly that the powder packing can be reproduced (note the fifth powder dependence on $D$ ). Secondly that the contact angle at the liquid-air-solid interface in a straight capillary has the same effect on capillary flow as at the complicated liquid-air-solid interface in a powder. Thirdly that Poiseuille flow in a straight capillary tube with a parabolic velocity profile corresponds to flow in a porous medium with a complicated pore structure. Despite the sweeping nature of these assumptions it must be said that the linear dependence of $M^{2}$ with $t$ is usually found in experiments, thus giving a certain validity to the practical application of the method.

It is also possible to derive an equation similar to 6(a) taking the pore structure in a bed of particles more directly into account in terms of the mean hydraulic diameter.

Hydraulic diameter $m=\frac{\text { volume of pores }}{\text { surface area of pores }}$

For a circular capillary tube $m=\frac{l \pi D_{\text {tube }}^{2} / 4}{l \pi D_{\text {tube }}}=\frac{D_{\text {tube }}}{4}$

For a bed of particles $m=\frac{\varepsilon}{(1-\varepsilon) S_{0}}=\frac{\varepsilon}{(1-\varepsilon)} \frac{D_{s v}}{6}$

The Laplace equation (Eq. (2)) for capillary pressure and Poiseuille's law (Eq. (10)) for fluid flow in a tube can also be expressed in terms of hydraulic diameter.

Laplace equation $P_{\text {capillary }}=\frac{4}{D_{\text {tube }}} \gamma \cos \theta=\frac{1}{m} \gamma \cos \theta$

Poiseuille's equation $\quad v^{*}=\frac{D_{\text {tube }}^{2} P}{32 \eta l^{*}}=\frac{m^{2} P}{2 \eta l^{*}}$

Here $\left(v^{*}\right)$ is the flow velocity in a capillary tube of length $\left(l^{*}\right)$ under a pressure drop P. Carman [10] has shown that if the pore structure of a bed of particles is taken to be represented as a bundle of capillary tubes then a correction should be made for the fact that the effective pore length between the top and the bottom of the bed $\left(l^{*}\right)$ is longer than the apparent pore length of the bed $(l)$. Furthermore the flow velocity in the pores $\left(v^{*}\right)$ is greater than the apparent flow velocity over the section of the bed $(v)$. Also as the pores in a bed of particles are not circular the constant two is replaced by a shape factor $k_{0}$. Thus substituting tortuosity $=\tau=l^{*} / l$ and $v=v^{*} \varepsilon / \tau$ in Eq. (10) gives:

$v=\frac{m^{2} \varepsilon}{k_{0} \eta} \frac{P}{\tau^{2} l}=\frac{m^{2} \varepsilon}{h_{k} \eta l}$

Carman grouped the shape factor and the tortuosity factor together as the Kozeny constant $h_{k}\left(h_{k}=k_{0} \tau^{2}\right)$. This constant has the role of linking the complexity of real pore space in a packed bed to the simple tubular geometry assumed in the derivation. It may be noted that if $k_{0} \approx 2.5$ and $\tau \approx \sqrt{2}$ then $h_{k} \approx 5$. In practice the Kozeny constant can be very different from five depending in the regularity of particle packing.

Expressing Eq. (11) in terms of volumetric flow rate $(q)$ given by multiplying the flow rate by the cross sectional area of the bed $(\Omega)$, i.e. $q=v \Omega q$ and $V$ is a liquid volume leads to: 
$q=\frac{m^{2} \varepsilon \Omega}{h_{k} \eta} \frac{P}{l}=\frac{d V}{d t}=\frac{\Omega d l}{d t}$

Integration leads to an expression similar to the Washburn equation.

$\frac{l^{2}}{t}=\frac{2 m^{2} \varepsilon P}{h_{k} \eta}$

Replacing pressure $P$ by the capillary pressure given by the Laplace Eq. (9) and substituting Eq. (8) for the hydraulic diameter of a bed of particles leads to the following:

$\frac{l^{2}}{t}=\left\lfloor\frac{\varepsilon}{(1-\varepsilon)} \frac{D_{s v}}{6}\right\rfloor \frac{2 \varepsilon \gamma \cos \theta}{h_{k} \eta}$

This can be expressed in terms of the mass of liquid taken up by the bed $M=\Omega \varepsilon l \rho_{l}$

$\frac{M^{2}}{t}=\frac{\varepsilon^{4}}{(1-\varepsilon)} \frac{D_{s v} \Omega^{2}}{3 h_{k}} \frac{\rho_{l}^{2} \gamma \cos \theta}{\eta}$

Comparing (15) with 6(b) shows that $C_{w}$ is given in terms of the characteristics of the powder bed as follows:

$C_{w}=\frac{\varepsilon^{4}}{(1-\varepsilon)} \frac{D_{s v} \Omega^{2}}{3 h_{k}}$

Furthermore it may be noted that the Kozeny-Carman equation gives the permeability $(B)$ of a packed bed as:

$B=\frac{\varepsilon^{3}}{(1-\varepsilon)^{2}} \frac{D_{s v}^{2}}{36 h_{k}}$

Thus $C_{w}=\sqrt{\frac{B \varepsilon^{5}}{h_{k}}} 2 \Omega^{2}$

It was indicated above that the Kozeny constant $h_{k}$ is used in permeability measurements to take into account the complexity of pore space in a real packing with respect to the assumed simplicity of the tube model. We propose a similar correction to Eq. (18) to try to take into account the complexity of capillary effects in powder beds with respect to the assumed simplicity of the capillary tube model.

Thus $C_{w}=h_{w} \Omega^{2} \sqrt{B \varepsilon^{5}}$

The derivation given above, based on effects in capillary tubes, indicates that the "Washburn" constant $h_{w} \approx 2 / \sqrt{h_{k}}$ or $\approx 0.89$. However, it is known that capillary effects in packed beds are dominated by the cellular nature of the pore space rather than by its "tubular" nature. This was demonstrated both theoretically and experimentally by Haines [11] and Hackett [5] in experiments on beds of spheres. They showed that passage of a liquid meniscus through a packed bed of particles does not occur as a regular smooth movement as in a capillary tube, but rather in sudden rapid movements called "Haines jumps" as successive volumes are invaded. The interconnected cellular nature of pore space also imposes a hysteresis in pressure/saturation changes such that desaturation takes place at a higher pressure than does re-saturation (or imbibition). Haines found the ratio between imbibition pressure and desaturation pressure was about $4.1 / 6.1=0.67$. All this suggest that $h_{w} \approx 0.89 \times$ $0.67 \approx 0.6$. The effects of these observation on the values of contact angle determined by the Washburn method, which is an imbibition procedure, will be discussed later in the paper.

It may also be mentioned that capillary rise must interact with the bed structure in a more complicated way than indicated by the analogy with capillary rise in tubes. For example the capillary rise in a conical tube with a taper of angle $\psi$ will not be governed by values of $\cos (\theta)+\psi$ but rather than by values of $\cos (\theta+\psi)$. Thus the contact angle determined by capillary rise cannot be identical to values determined by a sessile drop but must include some sort of bed structure parameter. The effect is masked by the essentially constant nature of bed structure imposed by the standard two sample method of applying the Washburn method.

\section{Materials and methods}

Preliminary experiments were performed with talc powder (Luzenac 00), with a mean particle size $D_{s v}=19 \mu \mathrm{m}$ as determined with a Blaine permeabilimeter (Prolabo SA), and true solids density of $\rho_{s}=2790 \mathrm{~kg} / \mathrm{m}^{3}$ as determined with an Accupyc 1330 helium pycnometer (Micromeritics SA). Further experiments were performed with five calcium carbonate powders (Durcal) supplied by Omya SA with volume mean particle sizes ranging from $5 \mu \mathrm{m}$ to $130 \mu \mathrm{m}$, and surface volume mean particles sizes from $2.4 \mu \mathrm{m}$ to $80.4 \mu \mathrm{m}$, as determined with a Malvern Mastersizer 2000 in dry powder mode. The true solids density was $2750 \mathrm{~kg} / \mathrm{m}^{3}$ as determined with the helium pycnometer. The liquids used were hexane: $\rho_{l}=655 \mathrm{~kg} / \mathrm{m}^{3}$, surface tension $\gamma=18.4 \mathrm{mN} / \mathrm{m}$. Water: $\rho_{l}=1000 \mathrm{~kg} / \mathrm{m}^{3}$, surface tension $\gamma=72.8 \mathrm{mN} / \mathrm{m}$. In the experiments with the calcium carbonate powders the water used was saturated with calcium carbonate and the surface tension measured to be $\gamma=73.4 \mathrm{mN} / \mathrm{m}$. All experiments were performed at the ambient laboratory temperature of $20^{\circ} \mathrm{C}$.

\subsection{Sample preparation}

As mentioned above, the key to applying the Washburn method is in obtaining two sample cells with two reproducible packings of the powder. The usual method is to fill the cells as carefully as possible and to tap each cell to reach the tightest packing possible. This can be very difficult to ensure especially for fine cohesive powders, such as talc, which do not settle well. Furthermore only one value of porosity, the tightest, is used thus making it difficult to test the validity of the theory.

Here we have prepared samples by a technique first proposed by Burt and Fewtrell [12] for preparing beds of powders for air permeability measurements. The method involves manual spatula filling of sample tubes with the powder under study and then compacting the beds by centrifugal force using a laboratory centrifuge. The method is extended here for both air permeability measurements and liquid capillary rise measurements. The centrifuge used in this work was a Hettich Rotofix 33 in which four samples were prepared in each run. It was found that the method gave four similar reproducible samples and that different packing porosities could be obtained for fine powders by changing the speed of rotation. The cylindrical sample cells length $80 \mathrm{~mm}$, wall thickness $1 \mathrm{~mm}$, inside diameter $17 \mathrm{~mm}$, were machined from one piece of aluminium with a base plate drilled with 69 holes diameter $0.8 \mathrm{~mm}$. A circular cut out of filter paper was placed in the bottom of the cell before filling with powder. A loose fitting PTFE piston is then put on top of the powder and four similar cells are loaded in the centrifuge (radius of rotation centre to bottom of the cells $=145 \mathrm{~mm}$ ) and set to rotate for $10 \mathrm{~min}$ at a fixed speed chosen between 500 and $4000 \mathrm{rpm}$. The position of the piston can be determined with a digital caliper to within $0.1 \mathrm{~mm}$ giving a good precision on the determination of the sample depth and hence the calculation of the porosity. The total volume of liquid taken up in a capillary rise experiment also gives another way of measuring the porosity of the samples.

\subsection{Air permeability measurements}

The air permeability of the powders packed by the method indicated above was measured. For this a continuous flow of dry air 
was passed through the beds and the flow rate and pressure drop measured using a mass flow meter (GFC 371, Analyt MTC, Colmar, France). Two mass flow devices were used with full range flows of $0-100 \mathrm{~L} / \mathrm{min}$ and $0-500 \mathrm{~L} / \mathrm{min}$ precision $\pm 1.5 \%$ full scale, reproducibility $\pm 0.5 \%$ full scale. The constant pressure used could be adjusted in the range $0-8$ bar. Generally a pressure of about 1 bar was used. The permeability was calculated using Darcy's law for compressible fluids (Carman 1956) where $\bar{p}=$ mean pressure and $\Delta p=$ pressure drop.

$B=q \frac{p_{1}}{\bar{p} \Delta p} \frac{\eta L}{\Omega}$

The mean permeability was determined from several different pressures runs for each sample. The experimentally determined air permeability of the samples were compared with the predictions by the Kozeny-Carman equation (Eq. (17)) by calculating the values of the Kozeny constant $h_{k}$. These results are given in Table 2.

\subsection{Capillary rise experiments}

Samples prepared by the centrifugal packing technique were used in capillary rise experiments performed with an automatic tensiometer (Model ILMS by GBX SA). This determines the weight of liquid taken up by the powder by capillarity as a function of time and the software of the instrument identifies the linear part of the $M^{2}$ versus $t$ curve and determines the slope. With each set of four sample cells prepared in the centrifuge, two capillary rise experiments were performed with hexane and two with water, a partially wetting fluid. Assuming that hexane is a perfectly wetting fluid with $\cos \theta=1$ the slope of the $M^{2}$ versus $t$ is used in Eq. (6b) to calculate $C_{w}$. This value of $C_{w}$ is then used with the slope of $M^{2}$ versus $t$ for water to determine the value of $\cos \theta$ and hence the contact angle $\theta$. In addition the porosity of the bed can also be determined by the amount of liquid taken up.

\section{Results and discussion}

\subsection{Talc samples}

Preliminary experiments were performed using talc powder to test the reproducibility of the centrifugal packing technique. These results are shown in Table 1. It can be seen that the porosity values determined from the bed dimensions ( $\varepsilon$ geometric) are slightly smaller than the values determined from the volume of liquid taken up ( $\varepsilon$ hexane) but do agree to within $2 \%$. It can also be seen
Table 1

Porosity of the talc powder packed at different speeds of rotation determined by the bed volume and by the volume of hexane imbibed by capillary rise.

\begin{tabular}{lllll}
\hline rpm & $\varepsilon$ Geometry & Std deviation & $\varepsilon$ Hexane & Std deviation \\
\hline 500 & 0.758 & 0.0133 & 0.759 & 0.0060 \\
1000 & 0.732 & 0.0071 & 0.744 & 0.0051 \\
1500 & 0.721 & 0.0044 & 0.730 & 0.0007 \\
1800 & 0.679 & 0.0071 & 0.692 & 0.0004 \\
2000 & 0.706 & 0.0052 & 0.715 & 0.0019 \\
3000 & 0.666 & 0.0164 & 0.684 & 0.0190 \\
4000 & 0.661 & 0.0057 & 0.678 & 0.0061 \\
\hline
\end{tabular}

from Table 1 that the packing porosity decreases as the speed of rotation increases showing that the centrifugal packing technique does allow preparing reproducible samples with a range of porosities, here in the case of talc powder the range is 0.66-0.76.

Typical results for capillary rise experiments are shown in Figs. 2a and b. Fig. 2a gives the basic results of mass of liquid taken up by capillarity as a function of time. Fig. $2 b$ shows the same results plotted as mass squared as a function of time where the linear section can be clearly identified and used with Eq. (6b) to determine the constant $C_{w}$. Fig. 3a shows the kinetics of capillary rise of hexane in talc samples packed to different porosities by being centrifuged at speeds from 500 to $4000 \mathrm{rpm}$. Fig. $3 \mathrm{~b}$ gives similar results for water. The slope of the linear part of these curves clearly depends on the porosity. The development leading to Eq. (15)

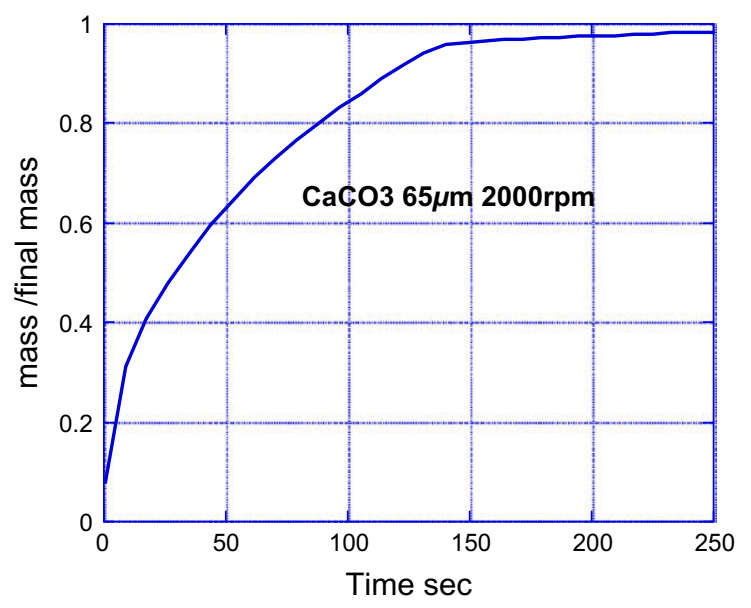

Fig. 2a. A capillary rise result, mass $M(\mathrm{~g})$ versus time (s).

Table 2

Results of the air permeability and the capillary rise measurements with calcium carbonate powders.

\begin{tabular}{|c|c|c|c|c|c|c|c|c|}
\hline Sample $D_{s v} \mu \mathrm{m}$ & $\mathrm{rpm}$ & $\varepsilon$ & $\mathrm{B} \times 10^{13} \mathrm{~m}^{2}$ & $h_{k}$ Eq. (17) & $C_{w} \times 10^{16}$ measured $\mathrm{m}^{5}$ & $\theta^{\circ}$ & $h_{w}$ Calculated & $\theta^{\circ}$ Eq. (19) $h_{w}=0.47$ \\
\hline Durcal 5 & 2000 & 0.560 & 0.206 & 7.05 & 6.51 & 77.1 & 0.62 & 73.0 \\
\hline \multirow[t]{2}{*}{$2.4 \mu \mathrm{m}$} & 3000 & 0.540 & 0.183 & 6.52 & 5.43 & 67.2 & 0.60 & 60.5 \\
\hline & 4000 & 0.528 & 0.154 & 6.88 & 4.60 & 62.7 & 0.58 & 55.1 \\
\hline Durcal 15 & 2000 & 0.499 & 0.898 & 4.63 & 7.77 & 64.7 & 0.47 & 64.7 \\
\hline \multirow[t]{2}{*}{$5.5 \mu \mathrm{m}$} & 3000 & 0.486 & 0.788 & 4.63 & 7.18 & 72.6 & 0.50 & 62.8 \\
\hline & 4000 & 0.478 & 0.621 & 5.42 & 5.28 & 69.1 & 0.43 & 70.6 \\
\hline Durcal 40 & 2000 & 0.483 & 1.60 & 3.29 & 7.96 & 65.7 & 0.39 & 69.9 \\
\hline \multirow[t]{2}{*}{$6.7 \mu \mathrm{m}$} & 3000 & 0.462 & 1.35 & 3.59 & 6.57 & 67.3 & 0.37 & 72.4 \\
\hline & 4000 & 0.447 & 1.36 & 2.68 & 6.74 & 63.7 & 0.44 & 65.7 \\
\hline Durcal 65 & 2000 & 0.443 & 2.78 & 4.95 & 9.61 & 63.4 & 0.46 & 63.8 \\
\hline \multirow[t]{2}{*}{$13.8 \mu \mathrm{m}$} & 3000 & 0.453 & 1.95 & 7.94 & 7.87 & 64.2 & 0.43 & 66.6 \\
\hline & 4000 & 0.448 & 1.81 & 8.46 & 7.53 & 59.7 & 0.43 & 62.8 \\
\hline Durcal 130 & 2000 & 0.471 & 59.3 & 11.1 & 50.1 & 65.7 & 0.44 & 67.6 \\
\hline \multirow[t]{2}{*}{$80.4 \mu \mathrm{m}$} & 3000 & 0.463 & 54.4 & 11.7 & 49.4 & 71.5 & 0.46 & 71.9 \\
\hline & 4000 & 0.466 & 75.4 & 8.5 & 54.2 & 72.3 & 0.43 & 73.6 \\
\hline
\end{tabular}




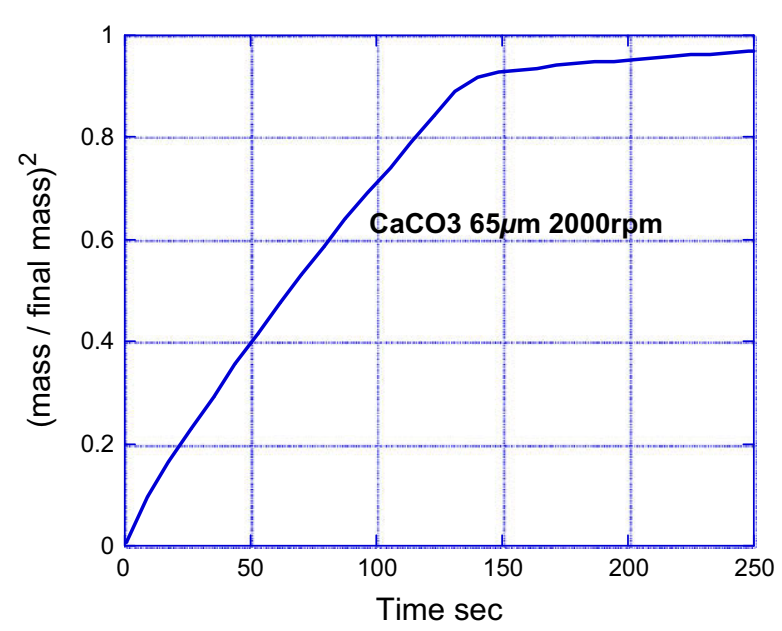

Fig. 2b. A capillary rise result relative mass $\left(M / M_{\infty}\right)$ squared versus time (s).

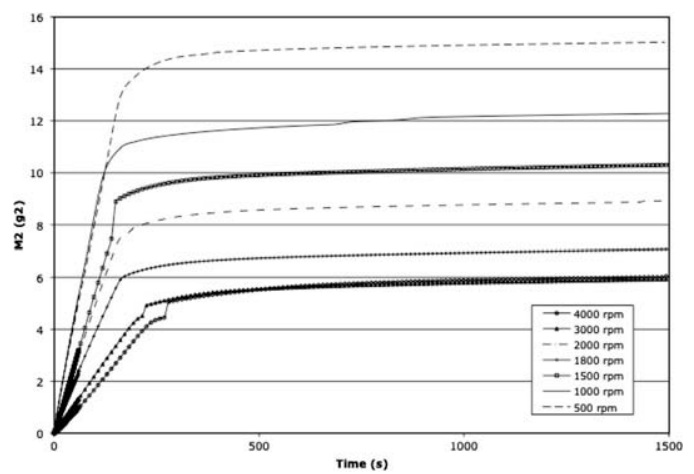

Fig. 3a. Kinetics of capillary rise of hexane in talc samples packed at different speeds of centrifugation (500-4000 rpm).

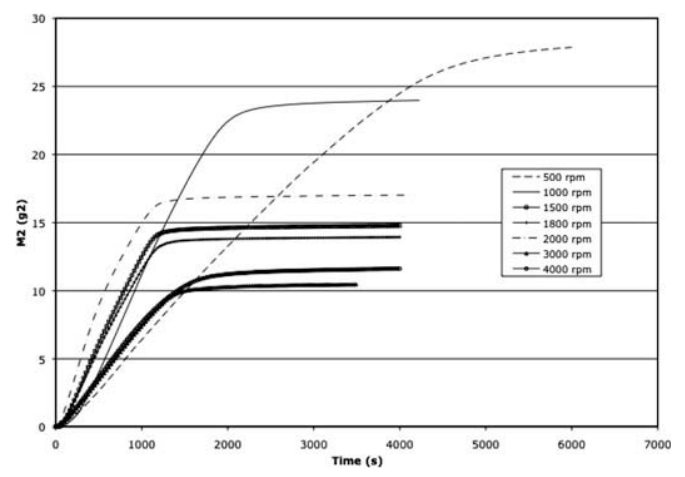

Fig. 3b. Kinetics of capillary rise of water in talc samples packed at different speeds of centrifugation (500-4000 rpm).

indicates that the variation of the rate of capillary rise with porosity depends on the function $\varepsilon^{4} /(1-\varepsilon)$. Fig. 4a shows the results of normalising Fig. 3a for hexane with this function. Fig. $4 \mathrm{~b}$ is the same applied to Fig. 3b for water. These figures indicate the validity of the porosity function which, despite the extreme sensitivity to the precision of the porosity values (note that it depends on the 4 th power of porosity), does bring the curves together.

Comparing these results for hexane and water also reveal that the porosity variations interact differently for a wetting fluid than for a partially wetting fluid. In particular the partially wetting fluid penetrates high porosity samples with more difficulty than does a

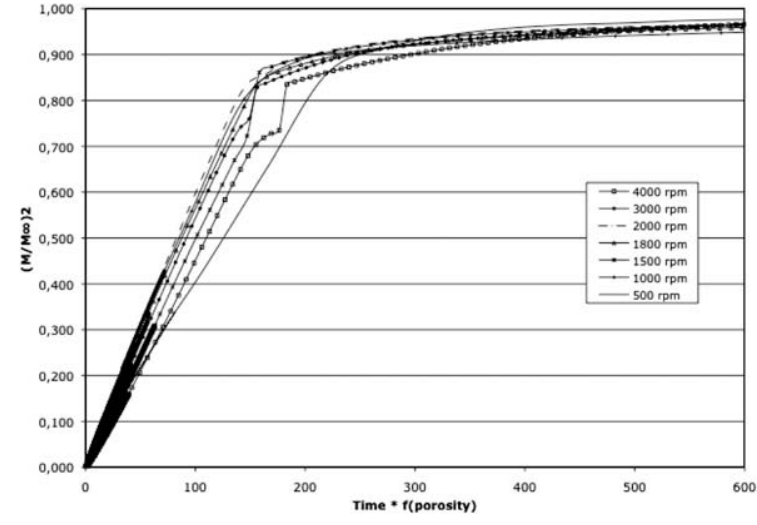

Fig. 4a. Results of Fig. 3a normalised using the porosity function.

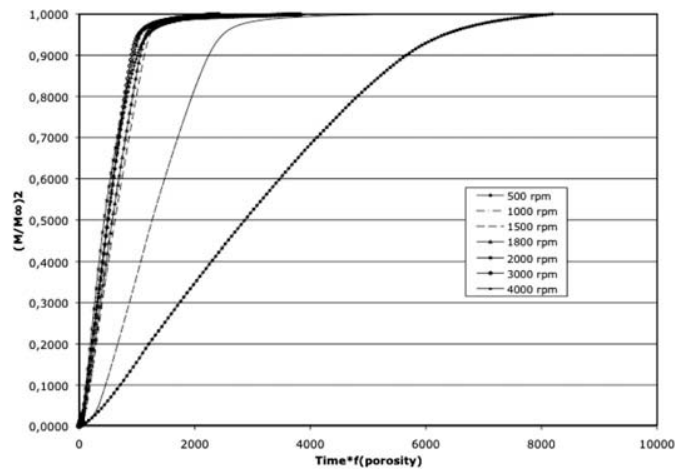

Fig. 4 b. Results of Fig. 3b normalised using the porosity function $\varepsilon^{4} /(1-\varepsilon)$.

wetting fluid. This is an indication that capillary effects in packed beds are more complicated than in a simple capillary tube.

Fig. 5 shows the water-talc contact angles determined for samples packed to different porosities. It can be seen that the values for the same powder vary from $83^{\circ}$ to $88^{\circ}$ as a roughly linear function of porosity in the range $0.66-0.76$. The greater the porosity the greater is the contact angle. The fact that the porosity function in Eq. (15) is successful in normalising the slopes of the $M^{2} / t$ curves emphasizes that the residual variation in contact angle with porosity is due to the geometry of the particle packing having other influences on the measurement of contact angle by the Washburn method.

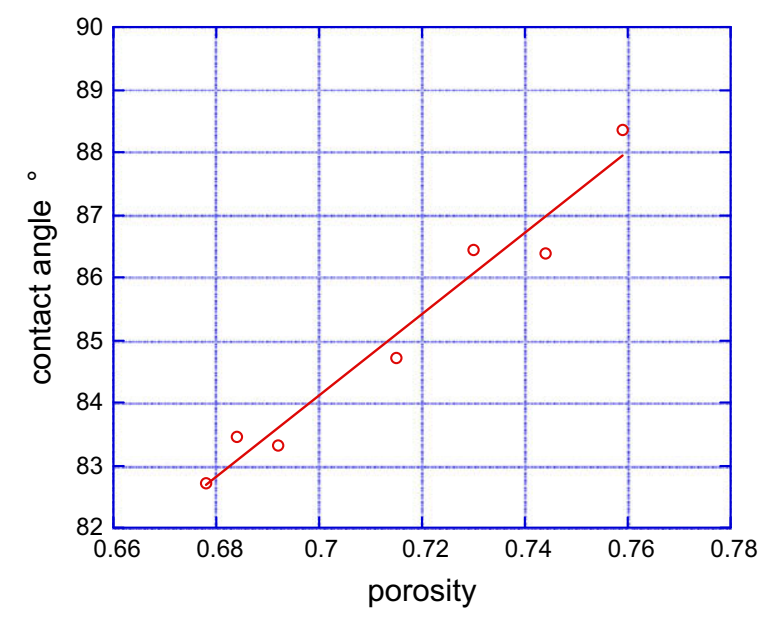

Fig. 5. Water-talc contact angles determined for different bed porosities. 


\subsection{Calcium carbonate samples}

Further experiments have been performed with calcium carbonate powders. These samples show a smaller variation in porosity with speed of rotation than do the talc samples but have the advantage of being available in a range of particle sizes allowing further examination of the method. Table 2 shows the overall capillary rise results and the air permeability values for the five powders, packed with three different centrifugal forces 2000, 3000 and $4000 \mathrm{rpm}$. The table gives mean values corresponding to 4-16 samples. The particle sizes are expressed as the surface volume mean diameter and range from $2.4 \mu \mathrm{m}$ to $80.4 \mu \mathrm{m}$ and porosities range from 0.44 to 0.56 . The porosity measurements have a standard deviation of about 0.003 , the permeability measurements have a standard deviation of about 0.2 and the final results of contact angle values have a standard deviation of about 0.01 .

Table 2 also shows the measured values of air permeability and illustrates the correspondence with the Kozeny-Carman equation by back calculation using the surface volume particle size determined by laser diffraction to obtain a value for $h_{k}$. Theory suggests that values of $h_{k}$ should be around five. It can be seen that values vary from about 3 to about 9 with a mean value of 6.3. This represents a reasonable correspondence for the Kozeny-Carman equation. Nevertheless the results for the $130 \mu \mathrm{m}$ sample are significantly higher than the others at about 11 , and may indicate a lack of precision in the mean surface volume particle size.

Table 2 also shows the values of the Washburn constant $C_{w}$ measured by capillary rise of fully wetting hexane. These values of $C_{w}$ are then used in Eq. (6b) with the results of capillary rise of water to determine the water-calcium carbonate contact angle. The values contact angle are seen to vary from $77^{\circ}$ to $60^{\circ}$ with a mean value of $67^{\circ}$. This indicates that water better wets calcium carbonate than talc. There seems to be a trend for a reduction in contact angle with increase in particle size but the variability of contact angle for each particle sample is too great to be affirmative.

The experiments with calcium carbonate powders have a much smaller variation of porosity with centrifugal force than those for talc powder, but examination does not confirm that the higher the porosity the higher the value of contact angle. The talc powders are found to pack to rather high porosities $0.66-0.76$ whereas the calcium carbonate samples pack to quite tight porosities $0.44-0.56$. These differences in porosity may be attributed to many features such as differences in particle shape and mechanical properties that affect flowability and the possibilities for particle rearrangements under packing forces. Comparing the contact angle results for talc and calcium carbonate results indicates that contact angle measurements are more affected by high porosity samples, which suggests that the influence of bed structure on capillary effects in packed beds is more complicated than can be taken into account by just porosity values. This could be masked by the standard sample packing method using only the tightest packing for ensuring reproducibly packed samples.

It was shown in the theoretical section that $C_{w}$ can be calculated from the experimental values of permeability using Eq. (19). Comparison of the results of this method with the results of the standard method for determining contact angle is made by back calculation to determine values of the factor $h_{w}$ in the same way that calculated values of the Kozeny constant $h_{k}$ were used above to check the validity of the values of permeability. Table 2 shows the values of $h_{w}$ calculated from Eq. (19) using the values of contact angles determined by the standard method. It can be seen that $h_{w}$ values vary from 0.37 to 0.62 with a mean value of 0.47 . As indicated in the theoretical section, an approximate value of $h_{w}$ is given by the expression $\left(2 / \sqrt{h_{k}}\right) \times(4.1 / 6.1)$. Furthermore, the experimentally determined mean value of $h_{k}$ of 6.3 would lead to a value of $h_{w} \approx 0.55$, not too different from the experimental values. This gives some confirmation of the validity of Eq. (19). Alternatively we can determine contact angles by using values of $C_{w}$ calculated from Eq. (19) using the experimentally determined values of permeability and $h_{w}$ taken to be the mean value of 0.47 . These results are also shown in Table 2 and serve to indicate that there is a reasonable correspondence with the values of contact angle determined by the standard method.

\section{Conclusions}

This paper presents a study of the Washburn method for determining the contact angle of liquids with powders. The standard Washburn equation has been modified to obtain expressions for the "geometrical" constant $C_{w}$ in terms of the parameters of the sample powder such as particle size and bed porosity. This is further extended by using the Kozeny-Carman equation to obtain an expression for the "geometrical" constant $C_{w}$ in terms of the permeability of the sample. This offers the possibility of eliminating the necessity for two identical packed samples of a powder to apply the Washburn method.

A novel method for preparing reproducible packed beds of powder using centrifugal force has been used to prepare samples for the Washburn method for the determination of contact angle between a liquid and a powder. Experiments with a talc powder of particle size $19 \mu \mathrm{m}$ with bed porosities in the range 0.66-0.76 show that values of contact angle determined by the Washburn method depend on the bed porosity; the greater the porosity the greater the value of contact angle.

Experiments with samples of calcium carbonate powders with particle sizes ranging from 5 to $130 \mu \mathrm{m}$ and bed porosities in the range $0.44-0.56$ do not indicate any significant variation of contact angle with particle size and porosity. Nevertheless it is shown that measurements of air permeability of the samples correspond to predictions of the Kozeny-Carman equation and that this can be incorporated in the Washburn equation to give a reasonable value of the Washburn constant $C_{w}$. It is therefore not necessary to prepare two identical samples for a determination of contact angle. A simple non-destructive measurement of bed porosity and air permeability can be used to obtain $C_{w}$ with here a mean value of 0.47 for the factor $h_{w}$.

The study indicates that the capillary rise of liquids in a packed bed of particles is more complex than would indicate the simple theory based on capillary rise of liquids in a tube system. It is suggested that the capillary model be further developed for irregular shaped capillary tubes.

\section{Acknowledgments}

The authors would like to thank the students Avni Jain from the IIT Delhi and Michael Hinchliffe from the University of Loughborough for their invaluable help with the experiments.

\section{References}

[1] T. Young, Philos. Trans. R. Soc. London, A (1805) 65-87.

[2] N.W.F. Knossen, P.M. Heertjes, Powder Technol. 20 (1965) 593-599.

[3] F.E. Bartell, H.J. Osterhof, Ind. Eng. Chem. 19 (1927) 1277-1280.

[4] J.A. Dodds, P. Srivastava, Part. Part. Syst. Char. 23 (2006) 29-39.

[5] F.E. Hackett, Disc. Faraday Soc. 17 (1921) 260-267 and 268-271.

[6] J.M. Bell, F.L.K. Cameron, J. Phys. Chem. 10 (1906) 658-674.

[7] E.W. Washburn, Phys. Rev. 17 (1921) 273-283.

[8] Y. Xiao, F. Yang, R. Pitchumani, J. Colloid Interface Sci. 298 (2006) 880888.

[9] N. Fries, M. Dreyer, J. Colloid Interface Sci. 320 (2008) 259-263.

[10] P.C. Carman, Flow of Gases Through Porous Media, Butterworth, London, 1956. [11] W.B. Haines, J. Agric. Sci. 17 (1930) 264-290.

[12] M.W.G. Burt, C.A. Fewtrell, The preparation of powder beds by a centrifugal compaction technique, in: M.J. Groves, J.L. Wyatt-Sargent (Eds.), Particle Size Analysis, Society for Analytical Chemistry, London, 1970, pp. 321-338. 